\title{
Linx
}

Revue des linguistes de l'université Paris X Nanterre

70-71| 2014

Variations sémantiques et syntaxiques : aspects d'une théorie de l'invariance

\section{Les auxiliaires modaux et l'inversion du sujet nominal en français et en anglais}

\section{Lucie Gournay}

\section{(2) OpenEdition}

\section{Journals}

Édition électronique

URL : http://journals.openedition.org/linx/1577

DOI : 10.4000/linx.1577

ISSN : 2118-9692

Éditeur

Presses universitaires de Paris Nanterre

Édition imprimée

Date de publication : 1 septembre 2014

Pagination : 165-183

ISSN : 0246-8743

Référence électronique

Lucie Gournay, "Les auxiliaires modaux et l'inversion du sujet nominal en français et en anglais », Linx [En ligne], 70-71 | 2014, mis en ligne le, consulté le 19 avril 2019. URL : http://journals.openedition.org/ linx/1577 ; DOl : 10.4000/linx.1577 


\title{
Les auxiliaires modaux et l'inversion du sujet nominal en français et en anglais
}

\author{
Lucie Gournay, Université Paris Est \\ EA IMAGER, UPEC, 94000 Créteil
}

\section{Introduction}

Cet article a pour objet la contrainte qui porte sur les auxiliaires modaux dans le cas particulier de l'inversion à sujet nominal. Par contrainte, je désigne la motivation qui est à l'origine des restrictions d'emplois de tel ou tel marqueur ou combinaison linguistique dans un contexte linguistique donné. Ces restrictions sont déductibles à partir de l'observation de corpus d'énoncés authentiques ou en ayant recours à des énoncés jugés non attestables. Par inversion à sujet nominal ${ }^{1}$, j'envisage les phrases simples, assertives, construites sur le schéma prédicatif: Groupe Verbal - Groupe Nominal Sujet. Celles-ci sont discutées dans la littérature (Bolinger 1977, Korzen (1988, 1996, 2006), Birner 1992, Dorgeloh 1997, Cornish 2001, Chen 2003, Marandin 2003, Gournay 2006, Lahousse $(2003,2011)$ inter alii), pour leurs propriétés informationnelles d'une part et pour leurs propriétés distributionnelles, qui sont exceptionnelles par comparaison à celles des phrases simples assertives sans inversion $\mathrm{du}$

sujet. 
(1) She was climbing a great hump of lava. Just beyond it were the tamarisks and the spring. (People ${ }^{2}, 83$ )

(2) Puis vint le moment où ils ne purent plus se défaire, plus revenir en arrière (...) (Jordan, 156)

La principale de ces propriétés exceptionnelles est la contrainte sur le sujet inversé : celui-ci se réalise uniquement sous la forme d'un GN lexicalisé : *Just beyond it were them/they; *Puis vint il/ lui. En ce qui concerne les propriétés informationnelles de l'inversion, on remarque que la valeur présentative est perçue comme typique de la forme (Le Bidois 1952, Bolinger 1977, Bailard 1981, Bresnan 1994 inter alii) sans pour autant être au fondement de cette forme (Birner 1994, Gournay 2009, Lahousse 2011 inter alii). L'entité exprimée par le sujet inversé (the tamarisks and the spring en (1), le moment où ils ne purent plus... en (2)) prend ainsi une valeur rhématique et l'on pose son existence comme nouvelle dans la scène verbale.

En anglais, la contrainte sur les auxiliaires modaux dans l'inversion à sujet nominal a fait débat et une question est posée : est-ce que (tous) les auxiliaires modaux sont attestables dans la phrase simple à sujet inversé ? Je répondrai en disant que l'on observe bien une contrainte en anglais et en français restreignant l'emploi des auxiliaires modaux dans l'inversion. Mais cette contrainte peut être qualifiée de relative dans la mesure où si la catégorie « auxiliaire modal» est attestée dans les deux langues dans l'inversion à sujet nominal, on observe une réduction de la variété des auxiliaires possibles et une réduction des modalités exprimées. Dans la perspective théorique dans laquelle ce travail se situe, ces restrictions sur les auxiliaires modaux sont à considérer comme les indices d'un comportement énonciatif qui caractérise l'inversion à sujet nominal étudiée.

Après quelques remarques $(\$ 1)$ sur l'adossement théorique, le corpus et sur la perspective comparée français / anglais adoptée ici, je rappellerai le caractère hétérogène de l'inversion à sujet nominal (\$2). Puis, je présenterai les restrictions sur les auxiliaires modaux comme elles ont été débattues pour l'anglais (\$3) et l'impact de leur prise en compte dans le développement d'une hypothèse sur la fonction informationnelle de l'inversion à sujet nominal. Considérant ensuite le français ( $\$ 4)$, je montrerai qu'une contrainte comparable à celle vue pour l'anglais peut être observée. Dans les deux langues, les restrictions sur les auxiliaires modaux dans l'inversion à sujet nominal doivent prendre en compte l'hétérogénéité des paramètres en jeu, allant des marqueurs (ici, les auxiliaires modaux, les types d'inversion) jusqu'aux types de discours $(\$ 5)$.

\section{Adossement théorique et précisions methodologiques}

Dans des travaux antérieurs, j'ai contribué au débat sur l'inversion à sujet nominal (Gournay 2006a, 2006b, 2009, Copy et Gournay 2009) en développant une approche énonciative constructiviste, comme on peut la voir définie dans Franckel (2002). Dans

\footnotetext{
2 Les références des exemples se lisent ainsi : le mot-clé du titre du roman (cf. bibliographie du corpus) et la page de l'édition consultée.
} 
ce cadre, le terme «constructiviste » implique que l'on entend étudier les fonctionnements énonciatifs dont on a des traces observables. C'est aussi l'idée qu'il y a des catégorisations construites dans les langues, qui sont plus complexes que celles qui sont accessibles intuitivement pour tout locuteur, via une reformulation du dire. L'objet du raisonnement linguistique est de faire émerger ces catégorisations faites dans les langues, en étudiant des données et en mettant au jour les compatibilités et incompatibilités entre marqueurs.

Pour ce faire, cette étude s'appuie sur un corpus d'exemples authentiques d'inversions à sujet nominal. Constitué à partir du dépouillement d'une vingtaine de romans contemporains ${ }^{3}$, il a été agrandi dans un second temps avec des exemples trouvés en lançant des requêtes ciblées sur internet : l'objectif de ce second temps de collecte était la vérification des données initiales.

Mon corpus final contient 350 occurrences pour l'anglais et 270 pour le français. Par rapport au corpus de Birner (1992) qui contient plus de 1700 occurrences $^{4}$, le corpus sur lequel je travaille est quantitativement réduit. Cependant, contrairement à Birner, qui s'intéresse aux valeurs informationnelles des éléments placés de chaque côté du verbe, mon approche se concentre avant tout sur les propriétés distributionnelles que je considère comme les indices d'un comportement énonciatif à identifier. Dès la première centaine d'exemples, des récurrences frappantes sont dégagées confirmant, ainsi qu'il est présenté dans la littérature, le caractère figé de la tournure et de son contexte d'apparition. À titre d'exemple, Le Bidois (1957) parle de 'tour', de 'locution figée' et Dorgeloh (1997, p.16) de 'strongly restricted devices'.

Le corpus collecté est ainsi conçu comme un échantillon représentatif des réalisations langagières. Certes, il n'est pas exhaustif d'un point de vue discursif puisque c'est un corpus largement littéraire, mais il révèle des compatibilités ou au contraire des incompatibilités dont on pose qu'elles ne sont pas dans le corpus par hasard. Ce qui est observé au niveau du corpus est révélateur des spécificités des langues étudiées. Lorsqu'une combinaison n'est pas attestée dans le corpus, c'est qu'il y a une restriction d'emploi à l'œuvre ; de même, lorsqu'une combinaison est très peu illustrée, par rapport à une autre concurrente (cf. l'alternance beyond it there was a spring vs. beyond it was a spring), c'est qu'une contrainte est probablement à l'œuvre. Les restrictions d'emploi sont les indices de fonctionnements linguistiques à identifier et ces derniers sont formulés en termes de déterminations énonciatives et prédicatives (Culioli 1990, 1999a, 1999b).

Si les restrictions observées sont les premiers outils de cette approche, l'interprétation joue néanmoins un rôle non négligeable dans le raisonnement, dans la mesure où c'est par l'interprétation que l'on détermine les valeurs sémantiques : par le biais de l'interprétation, on pourra, pour les auxiliaires modaux, déterminer par

3 Mes sources sont à l'origine uniquement littéraires et strictement contemporaines, à l'exception de Malraux, La Condition Humaine (1946). J'inclus des livres pour enfants (cf. bibliographie du corpus), des romans policiers et des romans divers. En dépouillant au total 19 romans, je collecte 100 exemples en français et 134 en anglais.

$4 \quad$ En anglais, Dorgeloh (1997) et Chen (2003) travaillent à partir d'un corpus d'occurrences réelles. Comme pour Birner, leur corpus est plus orienté discours journalistique. Dorgeloh rassemble 534 occurrences et Chen, 1200. En français, il existe quelques études sur corpus littéraires (Le Bidois 1957, Atkinson 1973) et plus récemment, sur un corpus diversifié, (cf. Lahousse (2003, 2011)). 
exemple leur capacité ou non à exprimer dans l'inversion à sujet nominal une modalité épistémique ou déontique.

Le fait de langue est abordé d'un point de vue contrastif, tant du point de vue du fait de langue lui-même, que du point de vue des traditions d'analyses. On passera donc de phénomènes d'inversion en anglais à des phénomènes d'inversion en français. Mais s'il y a de fortes ressemblances distributionnelles et stylistiques entre les deux langues sur ce point, il demeure du point de vue de la traduction qu'une inversion à sujet nominal en anglais ne donne pas forcément lieu, dans le passage vers le français, à la forme de phrase simple équivalente (et réciproquement). Il y a des restrictions observées et des contextes d'apparition qui sont spécifiques à chacune des deux langues.

La parenté des phénomènes d'inversion dans les deux langues est déjà bien établie dans la littérature, de sorte que les emprunts entre linguistes travaillant sur l'une ou l'autre langue sont fréquents. Bolinger 1977 emprunte pour décrire l'inversion en anglais l'analyse faite par Atkinson 1973 sur l'alternance en français entre Vint un bomme / il vint un homme. D'autres linguistes ont cherché aussi à comparer les phénomènes d'inversion entre les langues (Cornish 2001, Marandin 2003 inter alii) partant du constat que dans l'une comme l'autre il s'agit d'un agencement non canonique, réalisé dans une phrase simple. Néanmoins, malgré les emprunts et les parentés, il y a clairement, au sein des traditions linguistiques francophone et anglophone, des façons d'aborder le problème de l'inversion qui sont différentes : les contraintes syntaxiques sont au cœur du débat en anglais; c'est moins le cas en français, où ce sont plutôt les effets stylistiques ou informationnels qui sont débattus.

\section{L'inversion à sujet nominal : diversité et problématique de la fonction unique}

L'inversion à sujet nominal est un phénomène hétérogène, et selon les cas de figure, on parle dans une littérature, fondée en grande partie en français sur Le Bidois 1952, d'inversion locative, absolue, adjectivale, élaborative etc. Cependant, cette hétérogénéité est susceptible d'être minimisée par le fait que l'inversion dite locative est le cas de figure le plus fréquent, cf. (3) et (4):

(3) At the far end was a large window that let in the morning sun. (Red, 14) 5

(4) A côté étaient posés un haut-de-forme, une canne et une paire de gants. Jordan, 52)

On a, dans les deux langues, un groupe prépositionnel (GP) placé en tête de phrase (at the far end, à côté) et suivi directement d'un groupe verbal (GV) (was, étaient posés). Le GP dénote une localisation spatiale, d'où l'appellation d'inversion locative.

Comme autre type d'inversion à sujet nominal, on peut citer l'inversion absolue, caractérisée par l'absence de tout élément avant le groupe verbal (cf. 5), le non-accord

5 On notera que l'ordre canonique pourrait être rétabli sous la forme At the far end a large window that let in the morning sun could be seen mais ne pourrait être maintenu avec was comme GV: *At the far end a large window that let in the morning sun was. 
possible entre verbe et sujet en anglais, et au niveau des déterminations temporelles, le renvoi à une situation spécifique :

(5) Enter our protagonist, Frankie Paige... 6

Il y a aussi l'inversion de type adjectival (cf. 6), qui implique la présence d'un groupe adjectival en tête d'énoncé, constitué d'un adjectif faisant l'objet d'une reprise ou d'un degré :

(6) Mais, plus importantes encore sont les contributions des deux plus prestigieuses figures de la psychanalyse en Angleterre : Edward Glover et Ernest Jones 7 .

Il est nécessaire de garder à l'esprit la grande variété des formes d'inversion à sujet nominal, dont les exemples de (3) à (6) ne sont qu'un échantillon. Cette variété implique le déploiement de propriétés distributionnelles différentes d'un cas de figure à l'autre. Ainsi, le danger est de prêter à l'inversion dans son ensemble les singularités de fonctionnement de l'inversion locative (cf. 2 et 3) - qui est l'inversion la plus fréquente.

On peut prévoir que l'inversion locative et l'inversion absolue, qui se singularisent par un terme de départ déterminé spatialement (cf. le GP) pour l'une ou temporellement (cf. le GV) pour l'autre, n'auront pas les mêmes propriétés syntacticosémantiques que l'inversion adjectivale (cf. 6) dont le terme de départ qualitatif n'est pas déterminé en référence à une situation spatialement et temporellement définie. Selon la même logique, l'inversion locative, qui se fonde prototypiquement sur l'idée de perception (Atkinson $1973^{8}$, Bolinger 1977, Drubig 1988, Chen 2003, Dorgeloh 2006, Copy et Gournay 2009 inter alii) se distingue de l'inversion absolue qui fait appel à la représentation temporelle des événements.

Ceci ne signifie pas pour autant qu'il faille renoncer à trouver une motivation unique (informationnelle, discursive, ou autre) caractérisant toutes les formes d'inversions à sujet nominal, que ce soit en français ou en anglais.

Si dans les deux langues, l'inversion à sujet nominal est principalement discutée par les linguistes pour ses propriétés syntaxiques et stylistiques exceptionnelles, le but pour certains, dont Chen (2003) pour l'anglais, ou Marandin (2003) pour le français, est de faire le lien entre les propriétés distributionnelles et l'effet discursif produit. Pourquoi, par exemple, ne peut-on pas avoir les énoncés (7) et (8) ${ }^{9}$ ?

(7) * Out of the house was strolling my mother's best friend (ex 116 dans Chen 2003, qui l'emprunte à Coopmans 1989, p. 729)

(8) \# Il rentra dans le château. Dans le salon criait un groupe de femmes. (Marandin 2003, p. 48)

En (7), c'est une contrainte sur la forme progressive en BE+ING qui est signalée par l'étoile : dans l'inversion locative, l'aspect BE+ING n'est pas attesté. Cette

www.teako170.com/ps24.htlm

http://eric.bizot.pagesperso-orange.fr/desgros/klein/londres1.html

Atkinson (1973, p. 54) : "we have the illusion of being on stage and of seeing clearly with our own eyes".

9 Les jugements d'acceptabilité en* ou \# ont été affectés par les auteurs cités. 
contrainte, dont n'ont pas souvent conscience les locuteurs interrogés ${ }^{10}$, est vérifiée dans plusieurs corpus (Chen 2003, Gournay 2007) ${ }^{11}$, dans lesquels on constate que la forme BE + ING n'est pas attestée. En (8), le dièse devant la phrase signale une contrainte qui porte sur le verbe et qui selon Marandin (2003) est d'ordre pragmatique. Le verbe doit ainsi référer à une propriété attendue ${ }^{12}$ de sorte que Dans le salon papotait un groupe de femmes est pour l'auteur un énoncé plus acceptable que (8) mais aussi, peut-on conclure, que Dans le salon papotait un groupe d'bommes, l'action de «papoter» étant, pour l'auteur, prototypiquement associée aux femmes dans la situation décrite.

Les deux exemples de restriction observée en (7) et (8) nous rappellent, premièrement, que la restriction n'est pas forcément identifiée intuitivement, - c'est alors le dépouillement d'un corpus qui permet de la mettre en évidence - et deuxièmement, que la restriction peut être liée à un contexte très spécifique. D'autres restrictions sont signalées pour chacune des deux langues. En plus de celle du type de verbe (cf. 8) débattue en français et en anglais, et des restrictions aspectuo-modales (cf. 7), sont signalées des restrictions sur le sujet, sur la forme de l'élément antéposé non-verbal (quand il y en a) et sur l'interprétation générique de l'énoncé.

Dans mes travaux antérieurs, l'objectif, comme celui de Chen (2003) et de Marandin (2003), est de faire le lien entre une fonction énonciative de l'inversion et l'ensemble des propriétés distributionnelles remarquables qui la régissent. Je pose ainsi que les propriétés observées sont des indices d'un comportement énonciatif. Ceux-ci montrent que l'inversion à sujet nominal est une forme particulière de prédication ${ }^{13}$ qui a comme source énonciative une origine transindividuelle : au niveau de l'énoncé est encodée une source énonciative identifiée à une autorité qui transcende le point de vue singulier d'un locuteur; cette autorité est comparable, dans le cadre d'une analyse discursive, à l'hyper-énonciateur de Maingueneau (2004). Autrement dit, la source énonciative représentée dans l'énoncé n'est pas identifiable à un sujet humain, doué d'abstraction et de calcul.

La contrainte qui porte sur les auxiliaires modaux est l'un des indices les plus frappants de cette prédication qui ne laisse pas de place à l'expression d'une

10 "The progressive be, on the other hand, has not been found to occur together with the main verb, although native speakers quite readily accept such a structure (...) as grammatical. A tentative explanation will be proposed to explain this discrepancy between corpus counts and native speaker intuition. » Chen (2003, p.74)

11 Voir aussi sur cette contrainte, Quirk and al. (1972, p. 949) où il est noté que *There was standing our friend (avec un there accentué) est impossible à cause de la forme verbale en BE+ING alors que There stood our friend est tout à fait acceptable.

12 «Pour rendre compte de ces énoncés, j'admets une disposition narrative : tout protagoniste de récit ou de discours en tant qu'il est objet du discours ou de la narration a la capacité d'apparaître dans le discours ou d'en disparaître quand les circonstances (qui sont intrinsèquement liées au discours lui-même) ne l'empêchent pas. La capacité est attachée non pas au référent en tant que tel, mais au référent en tant qu'on le raconte. » Marandin (2003, p. 41)

13 Il y a "prédication », au sens employé dans cet article, lorsqu'une relation entre plusieurs éléments est construite dans l'énoncé en cours. On distingue ainsi la prédication dans « just beyond it was the spring» de la relation non prédiquée "the spring beyond it ». Ceci implique que la prédication est une relation complexe (construite par l'instanciation des variables d'un schéma de lexis) qui bénéficie des déterminations énonciatives de l'ensemble de l'énoncé (c'est-à-dire, de son ancrage dans une situation de discours spécifique par rapport à un sujet-énonciateur qui prend ou non en charge la prédication). 
subjectivité. L'auxiliaire modal, en français comme en anglais, est un marqueur qui est la trace d'une évaluation subjective, c'est-à-dire qui implique un calcul opéré par un sujet-énonciateur ${ }^{14}$. Le fait que l'apparition de l'auxiliaire modal soit contrainte dans l'inversion à sujet nominal montre l'incompatibilité entre un marqueur « subjectif » et un type de prédication qui se fonde, selon l'hypothèse ici, sur une origine énonciative transindividuelle. On verra, à partir d'exemples situés dans leur contexte, pourquoi cette incompatibilité n'est que relative et pourquoi il semble pertinent qu'elle ne soit pas cependant négligée.

\section{Contraintes sur les auxiliaires modaux dans l'inversion en anglais}

Coopmans (1989, p. 729) met en évidence plusieurs contraintes étoilées concernant l'inversion locative, dont celle illustrée ici :

(9)*Down the hill may roll the baby carriage. (Ibid)

En (9), on a un exemple d'inversion locative qui peut être comparé à l'énoncé attestable : Down the bill rolled the baby carriage. D'après Coopmans, c'est la présence du modal MAY qui rend l'exemple inacceptable. Cette contrainte est rediscutée par Chen (2003, p. 174) qui la réfute sur la base d'exemples authentiques trouvés dans son corpus. Toutefois, Chen ne prend pas en compte le fait que la remarque de Coopmans porte sur les cas d'inversion locative. Voici certains des exemples d'inversion avec auxiliaire modal que Chen cite :

(10) Even harder to manage would be a head trauma. (NY 3/13/00:69, Chen 2003, p. 175 ex. 122)

(11) Following the project description should be a selected list, not to exceed one page in length, of publications by other scholars and writers, or of primary material and sources that are especially pertinent to the proposed study. (NEH, Ibid, p. 176, ex. 129)

(12) The main thing was that there was no sense of proportion. I would chew a piece of gum at school, and the nun would say, "Jesus is very angry with you about that," and on the wall behind her would be a dying, bleeding guy on a cross. (NY 3/13/00:69) Ibid, p. 175, ex.121)

Dans les exemples qui réfutent la remarque de Coopmans, si tous les auxiliaires modaux de l'anglais sont envisagés (Chen 2003, p. 174-178), le lecteur ne trouve qu'un seul cas d'inversion locative concret faisant appel à un scénario de perception (cf. 12). Les autres exemples donnés sont des inversions non locatives et celles-ci ne mettent pas en jeu la représentation de la perception (cf. 10 et 11). Ainsi, Chen réfute pour l'inversion à sujet nominal la contrainte sur les auxiliaires modaux que Coopmans (1989) propose pour le seul cas particulier d'inversion locative. construits dans l'énoncé. Le sujet-énonciateur est un énonciateur doué d'évaluation (souhaitable / non souhaitable; possible / non possible etc.). 
Au sujet des auxiliaires modaux, Chen (2003, p. 178) remarque qu'il ne trouve jamais d'auxiliaire suivi d'un infinitif passé dans son corpus d'inversions à sujet nominal : il pose ainsi que la configuration MODAL+ HAVE -EN n'est pas attestée dans l'inversion à sujet nominal. Ceci signifie que selon Chen, la phrase *Following the description should bave been a selected list, not to exceed one page in length (ibid) illustre une contrainte absolue, qui porte sur la modalité quand elle exprime un irréel du passé.

Chen situe ses travaux dans le cadre cognitiviste. Il envisage les faits de langue comme des indices d'une conceptualisation, fondée sur le passage du concret au plan abstrait. Pour lui, l'inversion locative est le prototype concret dont sont dérivées des inversions plus figurées (inversions adjectivale, absolue etc.). Il a un point de vue que l'on peut qualifier de distancié sur le phénomène, puisqu'en dépit du recours au corpus et aux observables, il ne prend pas en compte l'hétérogénéité des cas d'inversion à sujet nominal, se fondant sur les postulats théoriques auxquels il adhère. In fine, après avoir réfuté la contrainte formulée par Coopmans (1989), Chen dégage une contrainte, qui porte sur la catégorie «auxiliaire modal» lorsqu'elle est associée à un paramètre supplémentaire, celui de la forme de l'infinitif.

Dans l'hypothèse qu'il formule, il se concentre sur l'expression de l'irréel qui va avec la combinaison Modal+Have-EN en anglais. Pour lui, l'irréalité non-immédiate (ibid) n'est pas compatible avec l'inversion parce qu'il y aurait une incompatibilité entre une prédication ayant comme point de départ un "ground », c'est-à-dire une donnée stable liée à la notion de "perceptual field» (ibid, p. 44) et la référence à l'irréalité non immédiate ${ }^{15}$.

À partir d'un corpus d'inversions strictement locatives, j’ai pu observer que la remarque de Coopmans (1989) était vérifiée dans une large mesure. Néanmoins, elle tolère ce que l'on pourrait appeler des exceptions. Est-ce que la présence de quelques exemples attestant la présence d'un auxiliaire modal suffit à lever toute idée de contrainte?

Dans le corpus constitué pour l'étude, sur 200 exemples authentiques d'inversion locative en anglais, on ne trouve que 3 énoncés avec des auxiliaires modaux. Ce qui est remarquable, en dehors du rapport de 3 sur 200 énoncés, c'est le type de modalité en jeu dans ces exemples. En effet, une seule configuration est attestée à trois reprises : COULD suivi du passif d'un verbe de perception non agentif (SEE et HEAR).

(13) Farther to the west could be seen a strip of blue where the ocean met Mission Bay, then just a lot of mud flats. ${ }^{16}$

(14) While the patient was lying on his back, just inside the apex beat, could be heard a queer crackling, bubbling sound with each systole. (Sapira's art and Science of Bedside Diagnosis, p. 343, Google book)

«...to direct the hearer's attention to a ground so that she can locate and subsequently focus on the figure - presupposes that there is a figure existing in the ground, which, as I discussed on 3.1., amounts to a contract that binds the speaker to present a figure once a ground is presented. But a modal+have structure fails to fulfil this requirement. Expressing a possibility or a hypothesis, the structure, if it occurred in inversion, would break the promise that once a ground is presented, a figure will follow. » (Chen, 2003, p.178)

16 www.sandiegohistory.org/ journal/83fall/gem.htm 
Dans ces exemples, on peut poser que le modal CAN à la forme passée suivi d'un verbe de perception apparaît dans son emploi le plus factif ou le moins virtualisant (Dufaye 2009). En effet, contrairement à ce que l'on aurait si COULD était suivi d'un verbe d'action comme come, avec COULD + verbe de perception passive, c'est une expérience de perception effective et non potentielle qui est exprimée. A ce propos, on peut rappeler les traductions de I could come qui correspond à je pourrais venir (conditionnel présent) et I could see qui correspond à je voyais / vis (indicatif imparfait ou passé simple). Ainsi, en (13) et (14) l'existence de a strip of blue ou de a queer crackling sound est assertée dans la situation représentée.

L'auxiliaire de modalité CAN dans les inversions locatives qualifie la perception en tant que capacité validée ; elle n'est pas envisagée dans le domaine plus abstrait des possibles. En ce sens, on peut parler ici d'un emploi assertif de l'auxiliaire modal CAN, qui ne renvoie plus alors au calcul d'un potentiel envisagé par un sujet-énonciateur. Renforçant cette hypothèse, on note l'importance d'un passif sans agent dans la combinaison. On représente là une entité susceptible d'être perçue par tous ; il ne s'agit pas d'une perception associée à un point de vue particulier mais d'une perception proche de celle exprimée par one could see dans :

(13') Farther to the west one could see a strip of blue...

Il existe d'autres occurrences exceptionnelles d'auxiliaires modaux, comme on le voit avec l'exemple de Chen (2003) déjà cité en (12) ou dans un exemple tiré de Levin et Rappaport Hovav (1996) :

(12) The main thing was that there was no sense of proportion. I would chew a piece of gum at school, and the nun would say, "Jesus is very angry with you about that, " and on the wall behind her would be a dying, bleeding guy on a cross. (Chen, p. 175, NY 3/13/00:69)

(15) In the garden may bloom the Christmas plant Joel Roberts Poinsett brought back from Mexico during his difficult ambassadorship there in the 1840s. (Levin et Rappaport Hovav 1996, p. 235, tiré d'une chronique publiée dans le Los Angeles Times)

Dans ces deux exemples, on représente des localisations stabilisées (on the wall; in the garden), temporellement vagues ${ }^{17}$, qui chacune convoque un ensemble de possibles avérés. En (12), on représente avec un would à valeur fréquentative des faits qui ont eu lieu de manière récurrente, telles des généralités ; ces faits récurrents illustrent ainsi ce qui est argumenté dans le contexte : "People talk about how horrible it is to be brought up Catholic, and it's all true. The main thing was (...)». En (15), dans cette chronique listant les éponymes célèbres qui occupent l'espace quotidien des californiens, on réfère à une plante qui se trouve immanquablement dans tous les jardins. Les anglophones interrogés sur cet énoncé remis dans son contexte ${ }^{18}$ ont

17 Dans la grande majorité des exemples d'inversion locative, le contexte d'une situation temporelle spécifique est construit dans le contexte gauche.

18 Voici l'extrait en contexte : In the kitchen cupboard or refrigerator or at ladies' socials, we find the works of the late Earl S. Tupper, a chemist with DuPont who was fascinated with the properties of polyethylene. In the living room ashtray may be the remains of Jean Nicot's eponym. A French diplomat in Portugal in the 1560s, Nicot received a smokable plant from the New World, which his grateful sovereign named Nicotiana tabacum and the U.S. Surgeon General later condemned as nicotine. 
évoqué l'impression d'un «imaginary guided tour » pour reprendre la formulation de Drubig $(1998$, p. 87), qui peut se répéter. Ainsi, comme pour l'exemple (12), on a un emploi particulier de l'auxiliaire modal, qui réfère à une situation fictive présentée avant tout comme avérée, et susceptible de se répéter: on exprime une propriété récurrente pour un repère de lieu donné.

En dépit de l'apparition de tels exemples, il semble possible de dégager une restriction d'emploi formulable en ces termes :

Dans les inversions locatives en anglais, on trouve peu d'auxiliaires modaux. Ceux qui sont susceptibles d'apparaitre, les auxiliaires comme COULD, WOULD ou MAY, voient leur champ de valeur réduit, du fait du contexte inversé, et ce sont leurs emplois les plus factifs (perception effective, valeur fréquentative et sporadique) qui émergent.

Dans les inversions à sujet nominal en anglais, la configuration auxiliaire modal + infinitif passé est une contrainte absolue. (Chen, 2003)

Il y a au travers de cet ensemble de restrictions, une cohérence énonciative qui permet, avec d'autres restrictions observées, de cerner le type de prédication en jeu dans l'inversion locative et les autres formes d'inversion à sujet nominal. On voit qu'un auxiliaire modal qui suppose la croyance d'un sujet-énonciateur singulier n'est pas envisageable. Seule une modalité renvoyant au constat impersonnel ou au savoir universel est compatible avec la tournure inversée. Avec l'inversion locative, la configuration de ce constat est largement liée à la représentation de ce qui peut être perçu par tous.

\section{Existe-t-il, à l'instar de l'anglais, des contraintes sur les auxiliaires modaux dans l'inversion en français?}

Dans la littérature sur l'inversion à sujet nominal en français, il n'y a pas de débat explicite sur la compatibilité ou incompatibilité des auxiliaires modaux. J'argumenterai qu'il y a lieu de dégager une telle incompatibilité, même si celle-ci doit être relativisée en fonction des types d'inversion et des critères contextuels spécifiques.

Dans les références consultées, en dehors de la contrainte sur le type de verbe, dont on a vu un aspect en (8), il n'y a pas de contrainte absolue ni relative relevée dans le groupe verbal.

Marandin $(1999,2003)$, qui se focalise sur les inversions locatives et absolues, annonce qu'il met de côté les contraintes syntaxiques et ne retient que les contraintes d'ordre informationnel ou pragmatique. Contrairement à Chen par exemple, Marandin ne lie pas les éventuelles contraintes syntaxiques à un comportement informationnel ou pragmatique de la forme.

Dans Lahousse $(2003,2011)$ - une étude sur le sujet post-verbal en français moderne, qui contrairement à celle de Marandin se fonde sur un corpus d'exemples

In the garden may bloom the Christmas plant Joel Roberts Poinsett brought back from Mexico during his difficult ambassadorship there in the 1840s. (Extrait de Eponyms Bequeath a $2^{\text {nd }}$ Life on Names, by Sid Moody, paru dans le Los Angeles times, 2 décembre 1990) 
authentiques - si plusieurs contraintes portant sur le groupe nominal sujet sont repérées et discutées, aucune portant sur le groupe verbal n'est discutée.

À propos de l'inversion locative, Fuchs (2006) rappelle, en les illustrant d'énoncés fabriqués étoilés, les contraintes sur le régime du verbe, qui ne peut pas avoir de complément d'objet. Elle signale aussi que l'ordre inversé a tendance à privilégier un groupe verbal court et peu déterminé du point temporel et modal. La tendance énoncée par l'auteur ne fait pas l'objet d'une illustration par des énoncés fabriqués étoilés, et demeure par conséquent imprécise.

Il est pourtant possible de montrer qu'il y a aussi une restriction d'emploi sur l'apparition des auxiliaires modaux dans l'inversion à sujet nominal en français.

Dans la perspective d'un parallèle avec ce qui a été vu pour l'anglais, une première étape serait d'étudier la combinaison inversion locative et auxiliaires modaux (cf. remarque de Coopmans (1989) discutée en 3.).

Dans mon corpus, qui contient 170 exemples d'inversions locatives en français, il n'y a aucun exemple de GV comprenant un auxiliaire modal. Cette observation, qui va de pair avec l'absence de négation dans le $\mathrm{GV}^{19}$, semble mettre en évidence non pas une préférence comme signalée dans Fuchs 2006, mais une contrainte. Dans un premier temps, on peut tenter d'illustrer la contrainte de la manière suivante :

(16) ?* A côté de l'écran devrait/devait se trouver un projecteur multicolore.

La contrainte illustrée en (16) est évaluée comme forte (cf. l'indice «?*»). Dans la perspective énonciativiste, elle rend compte du fait qu'il est peu probable, comme constaté dans le corpus, de combiner une modalité déontique ou épistémique avec une inversion locative.

On peut opposer à (16) un énoncé attestable plus canonique comme par exemple à côté de l'écran on devait trouver / devrait / aurait dû trouver un projecteur multicolore. La taille du corpus, sa particularité (un corpus littéraire) et l'absence de discussion de cette contrainte dans la littérature antérieure francophone appelle néanmoins à une certaine prudence.

Une façon de montrer qu'il existe bien une contrainte comme celle illustrée en (16) consiste à avoir recours à la falsification. Ici, cela implique donc de trouver des énoncés authentiques qui illustrent la combinaison inversion à sujet nominal et auxiliaires modaux.

Lorsque l'on cherche sur Frantext ou Google les suites « se trouver un(e) », on obtient tout de suite des exemples d'inversion inclus dans une proposition subordonnée en où :

(17) Il évita, stupéfait, le méhari traînant sa bosse de par les rues et s'approcha d'un barbu affalé sur un sommier métallique jeté là où aurait pu se trouver un trottoir. (Frantext, FALLET René, Le Triporteur, 1951, p. 136)

(18) Au bout de quelques minutes, Serge disparut derrière le paravent où devait se trouver une desserte, car Élisabeth l'entendit remuer des couteaux et des fourchettes dans un tiroir. (Frantext, GREEN Julien, Minuit, 1936, p. 226)

19 L'absence de négation dans le GV de l'inversion à sujet nominal est aussi constatée en anglais et débattue par de nombreux auteurs (Dorgeloh 1997, Chen 2003, Gournay 2006a inter alii). 
En (17) et (18), l'apparition de l'auxiliaire modal va de pair avec la nature subordonnée (proposition relative ou circonstancielle) de la phrase inversée. Ces exemples vont dans le sens de ce que remarquait Atkinson (1973) : la nécessité de distinguer l'inversion à sujet nominal dans la phrase simple de celle qui apparaît dans la phrase subordonnée. En effet, pour Atkinson (1973, p. 21), le corpus sur lequel est fondée son analyse montre que les contraintes sur les verbes sont plus fortes avec l'inversion dans la phrase simple et que l'alternance avec la structure impersonnelle en Il n'est pas non plus de même ordre. Cette nécessité de distinction, ainsi que la neutralisation de la contrainte illustrée en (16) dans les exemples en où AUXMOD se trowver un(e) X sont des éléments à retenir pour argumenter, comme Atkinson (1977), en faveur d'une distinction en français entre l'inversion dans une proposition subordonnée et l'inversion dans la phrase simple ${ }^{20}$.

Si les exemples (17) et (18) font émerger une inversion à sujet nominal qui d'un point de vue énonciatif n'est pas du même ordre que l'inversion locative étudiée dans cet article, il existe une autre famille d'exemples - moins importante - qui doit malgré tout être prise en compte.

De fait, d'après les exemples collectés par sélection sous Google ${ }^{21}$ ou Frantext, on constate que l'auxiliaire modal n'est pas rare. Il apparait au sein d'une phrase simple à inversion du sujet nominal dans un contexte discursif identifiable: celui d'une reconstitution historique ou d'événements. ${ }^{22}$

(19) Toutes les tours étaient couvertes d'un toit. De même, les chemins de rondes étaient eux aussi couverts d'un toit (si l'on se fie à la vue contenue dans les albums des ducs de Crö̈). Au XIXème siècle, seule la tour d'angle (plan en E) était encore couverte d'un toit (d'après dessin de Vasseur). Caché derrière le châtelet, devait se trouver un bâtiment servant à abriter les communs. ${ }^{23}$

On remarque dans ce cas de figure que le potentiel de valeur de l'auxiliaire modal est réduit : la modalité déontique n'est pas attestée et, en ce qui concerne la modalité épistémique, il semble qu'elle puisse porter sur la prédication d'existence de l'entité, à condition d'exprimer une quasi-certitude vérifiée par des indices objectifs. C'est ce qu'on peut voir en (19) et dans la sélection d'exemples ci-dessous :

20 Cette distinction peut être motivée, d'un point de vue énonciativiste, par la différence de prédication en jeu. La proposition subordonnée fait état d’une prédication envisagée comme repérée par rapport à la situation d'énonciation : elle est conçue comme non nouvelle ou accessible pour le co-énonciateur; la proposition principale fait état d'une prédication repérée par rapport à l'énonciateur et qui est conçue comme nouvelle pour le co-énonciateur. De sorte que l'inversion dans une subordonnée est soumise à des repérages énonciatifs bien différents de ceux de l'inversion dans la phrase simple, ce qui influe sur l'effet discursif et les propriétés distributionnelles.

21 À titre indicatif, voici les résultats de la recherche "devait se trouver le» : sur 295 exemples, il y a $70 \%$ d'inversions en contexte subordonné (" où / lequel devait se trouver le... »), 20\% d'occurrences dans des énoncés non inversés (" elle devait se trouver le lundi $8 . . . »)$, et $10 \%$ d'inversions en proposition indépendante. Parmi ces dernières, $7 \%$ apparaissent dans des inversions locatives, $3 \%$ s'avèrent être des cas d'inversion avec un GP exprimant une localisation figurée (" $A$ sa tête devait se trouver le seigneur de la guerre... »).

22 Afin de disposer du contexte large, j'ai recours ici aux exemples collectés sur Google ; les contextes accessibles sous Frantext sont trop limités. Plusieurs requêtes ont été lancées. A titre d'exemple, la requête " pouvait se distinguer un(e) » donne sur Google 13 exemples dont 3 seulement illustrent les inversions à sujet nominal étudiées.

23 http://users.skynet.be/genehist/brifr.Htm 
(20) L'église fut commencée en 1097 et consacrée dans la nuit de Noël 1100. La nef a aujourd'hui disparu, peut-être effondrée lors de sa construction. Ce prieuré était organisé d'une façon classique avec l'Eglise au Nord, orientée Est-Ouest, le cloître se trouvant vers le Sud, organisé en quadrilatère dans l'axe Nord-sud. Il prenait appui à l'Est sur le bâtiment comprenant la salle capitulaire, surmontée du dortoir des chanoines relié par un escalier au transept droit; au Sud devait se trouver le scriptorium, où les chanoines faisaient des travaux d'écriture. Sur le site se trouvent également à l'ouest les restes d'une tour fortifiée du XII ${ }^{\text {ème }}$ suivie d'une grande salle voûtée. Ce bâtiment devait servir de salle forte pour la population. ${ }^{24}$

(21) Il y eut un échange verbal d'une violence extrême entre les deux protagonistes. L'infirmière hurlait. Ses cris répondaient en écho aux vociférations du médecin hors de lui. Les autres témoins de la scène devaient se tenir en silence à l'ombre d'eux-mêmes. Je les imaginais tapis dans un coin de la pièce. Ils laissaient alors face à face le médecin et l'infirmière ; entre les deux, devait se trouver le malade dont le destin se jouait à ce moment-là précisément. ${ }^{25}$

Dans les exemples (19) à (21), le sujet postverbal réfère à une entité dont l'existence est envisagée comme quasi-certaine à partir d'indices réels. Il s'agit à chaque fois d'une reconstitution de lieu pour (19) et (20), de scène pour (21). Si l'on prend en compte le contexte, on constate que l'existence de l'entité repérée (le bâtiment en (18), scriptorium en (19), le malade (20)) est calculée à partir de faits constatables ou de sources extérieures à la démarche déductive d'un sujet-énonciateur.

En (19) il s'agit de la reconstitution d'un château à partir de plans / dessins ; le texte commente un plan du château qui est donné sur le site. Ce n'est d'ailleurs pas l'existence du bâtiment qui est remise en question, mais sa fonction (cf. "servant à abriter les communs ») qui est évaluée comme quasi-certaine (DEVOIR) selon les sources disponibles.

En (20), après l'inversion locative signalée en gras, on trouve la prédication non modalisée «Sur le site se trouvent également à l'ouest les restes d'une tour... », avec l'adverbe également qui renvoie à la prédication d'existence du scriptorium, dans l'inversion locative qui précède. Une distinction est exprimée entre la prédication d'existence de ce qui est visible (cf. les restes) par rapport à la prédication d'existence de l'entité elle-même, qui n'est plus visible (cf. le scriptorium), mais dont l'existence est néanmoins posée dans le plan décrit.

En (21), il s'agit d'un témoignage, dont la fonction est de convaincre de la validité de ce qui est représenté. On a ici un aperçu du va-et-vient possible entre ce qui est subjectif (cf. "Je les imaginais tapis dans un coin de la pièce ») et ce qui est constatable par tous (cf. "Les autres témoins de la scène... »). Cet exemple peut être comparé à un autre type de prédication d'existence : « entre les deux, il devait y avoir le malade dont le destin (...)». Dans les deux cas, on a l'auxiliaire DEVOIR, avec une valeur épistémique puisque la présence du malade «entre les deux » est fortement probable. Mais la prise en charge un sujet-énonciateur n'est encodée que dans l'énoncé manipulé («il devait y avoir...»), ce qui a pour effet d'exprimer une modalité 
épistémique à partir d'une reconstitution subjective que l'on pourrait gloser « d'après ce que j'imaginais». Dans l'énoncé original, la combinaison de l'inversion et de l'auxiliaire modal semble exprimer une reconstitution objectivée que l'on pourrait cette fois gloser par «d'après ce qui a été dit». Cette reconstitution objectivée fait écho à l'effet «caméra », déjà signalé dans la littérature sur l'inversion (cf. 'displaced immediacy', Dorgeloh, 1997, 2006).

Dans tous ces cas, l'auxiliaire modal DEVOIR apparaît dans l'inversion à sujet nominal dans des contextes spécifiques, pour lesquels il est possible de parler de reconstitution in vivo de la réalité. La valeur épistémique de quasi-certitude est endossée par des sources patentes ou des preuves factuelles, extérieures au point de vue subjectif d'un sujet-énonciateur ou présenté comme tel.

\section{Le paramètre du type de discours}

Comme on l'a vu, il est nécessaire de faire varier les types de textes pour s'assurer de la validité d'une contrainte : le corpus d'origine littéraire ne contient pas d'inversion locative composée d'un auxiliaire modal; la recherche sur Frantext et Google permet d'en collecter, dans des extraits descriptifs non littéraires. Avec les exemples (19) à (21), on voit qu'il y a une corrélation évidente entre la fonction du discours - reconstituer quelque chose qui passe pour la réalité - et les compatibilités qu'il permet.

On vient de voir qu'il existe en français une contrainte relative, mais significative dans notre démarche, sur l'apparition des auxiliaires modaux dans l'inversion locative au sein d'une phrase simple. Selon le point de vue adopté ici, il est posé que l'incompatibilité décrite provient d'une incompatibilité de repérages énonciatifs et non d'une incompatibilité de catégorie syntaxique.

Qu'en est-il du phénomène plus général d'inversion à sujet nominal ? Peut-on, comme pour l'inversion locative, parler de contrainte en anglais et en français ?

Pour l'anglais, Chen (2003) produit des exemples d'inversion à sujet nominal qui comprennent des auxiliaires modaux. Il reste à déterminer, cependant, si des conditions d'apparition particulières rendent licites ces apparitions d'auxiliaires modaux. Est-ce que seules certaines valeurs modales apparaissent? Est-ce qu'un contexte discursif spécifique doit être mobilisé ?

Les exemples d'inversion à sujet nominal comprenant un auxiliaire modal recueillis pour cette étude (ceux donnés dans Chen (2003) et ceux de mon corpus) partagent une propriété récurrente : la modalité exprimée dans l'inversion est frayée dans le contexte antérieur ; c'est-à-dire que la modalité exprimée dans l'inversion fait l'objet d'une reprise.

(22) Bowerbox believes that significant trauma to a limb, for example, would probably be treated with immediate amputation rather than arduous repair. Even harder to manage would be a head trauma. (NY 3/13/00:69, Chen 2003, p. 175, ex. 122))

(23) In 1983, they all will be gone: William G. Milliken after 13 years in Michigan, James A. Rodhes after sixteen years in Ohio, Robert D. Ray after 14 years in Iowa. Gone also will be Minnesota's Albert Quee (...) (Birner 1996, p. 56, ex. 1-a, cité par Chen, 175, ex. 120) 
(24) As those become more global, two things could happen: First could come displacement -- a U.S. job goes elsewhere. Then could come the secondary effect of a U.S. labor surplus in a variety of highly skilled professions. ${ }^{26}$

Dans les exemples (22) à (24), on remarque des spécificités communes, qui pourraient servir de pistes à travailler. L'auxiliaire modal (ici, WOULD, WILL, COULD) qui apparaît dans l'inversion fait écho à une première occurrence de modalité posée dans le contexte antérieur. Comme les exemples sont rares, il est difficile à ce stade du corpus de dire s'il s'agit d'une tendance ou non, mais on remarque aussi que dans tous les cas, l'inversion est intégrée dans une énumération. Ce genre de propriétés, en relation avec la construction de la cohésion discursive, doit être pris en compte.

En ce qui concerne le français, il existe une forme d'inversion complète qui ne semble pas subir la contrainte des auxiliaires. Il s'agit de l'inversion appelée élaborative dans Marandin (2003). Cette inversion en verbe-sujet, soumise à la contrainte d'une interprétation générique, se rencontre dans les textes qui font état de vérités générales ou de lois.

(25) Ensuite peuvent survenir des troubles digestifs (diarrhées), respiratoires (toux, pneumonie), cutanés (pustules, croissance anormale de la peau)...27

(26) Ne peut être considéré comme étant à bonne maturité tout lot unitaire de vendange présentant une richesse en sucre inférieure à 180 grammes par litre de moût pour les vins rouges et rosés et à 170 grammes par litre de moût pour les vins blancs. ${ }^{28}$

(27) Le 20 mars 1793, le territoire du district demande le contingent du complément de l'armée montant à 44 hommes. Le tirage est fixé au 22 mars ; devront se présenter tous les célibataires et veufs sans enfants de 16 à 40 ans. Le 22 mars, 283 citoyens sujets au tirage se présentent. Voici les formalités : «Avons mis dans un chapeau pareil nombre de billets, dont 44 noms pour désigner les soldats. En conséquence, la voie du sort nous a fournis pour marcher à la défense de la patrie... ».29

Ainsi, dans ces inversions que l'on trouve dans des textes à fonction explicative ou législative, la modalité remplit un rôle cohérent par rapport au genre de discours. En (25), on présente une maladie en fonction d'un ensemble de symptômes potentiels (cf. «valeur sporadique »), avérés par l'autorité scientifique universelle. En (26), il s'agit d'un extrait de décret, texte qui par essence définit les obligations, et les interdictions de manière objective. L'auxiliaire modal POUVOIR a ici une valeur déontique. On remarque que la négation, qui est incompatible avec d'autres types d'inversion à sujet nominal (Chen 2003, Gournay 2006), est possible ici pour les mêmes raisons : l'origine énonciative représentée est une autorité dont sont issues les interdictions, les devoirs et les permissions. Ainsi, il y a compatibilité entre d'une part les modalités discursives en jeu et l'expression d'une modalité déontique prise en charge par une source

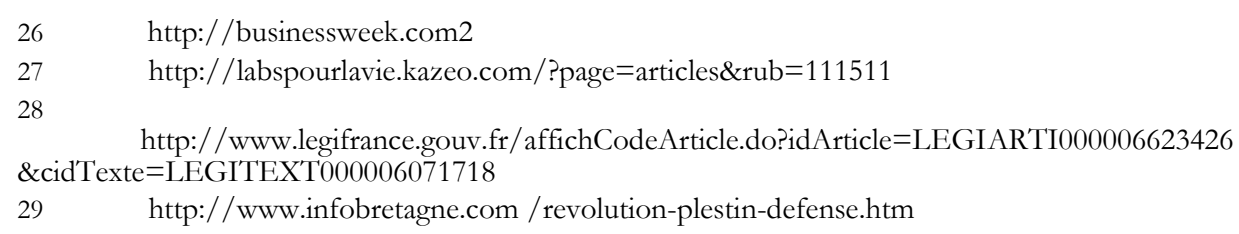


énonciative non individuelle. Enfin, dans l'exemple (27), l'inversion élaborative rend compte d'un règlement. Dans le contexte antérieur immédiat, on remarque un énoncé au passif sans agent (cf. «est fixé ») : la précision sur la date est exprimée en dehors de toute mention d'une source décisionnelle. De même, l'inversion élaborative renforce le repérage par rapport à une autorité qui dépasse l'évaluation d'un sujet-énonciateur.

\section{Conclusion}

Il existe probablement d'autres cas de figure récurrents, dans d'autres types de discours, qu'il serait intéressant d'identifier et d'analyser. Malgré l'aspect parcellaire des exemples collectés, il me semble pouvoir justifier que l'apparition des auxiliaires modaux dans l'inversion à sujet nominal est soumise à un ensemble de contraintes énonciatives ou du moins des conditions qui assurent la cohérence des paramètres discursifs du propos. Ainsi, l'auxiliaire modal est attesté dans l'inversion à sujet nominal, en anglais et en français, quand les conditions contextuelles et discursives lui permettent d'exprimer une modalité qui ressort d'une évaluation qui est non subjective. Dans le cas particulier de l'inversion locative, cette évaluation d'origine transindividuelle peut être identifiée à des signes factuels (cf. ce qui est constatable par tous dans la situation décrite) ; avec les inversions élaboratives, la modalité déontique est associée à la représentation d'une autorité construite dans le discours en cours (cf. un règlement, une loi) et non à l'évaluation d'un sujet-énonciateur.

Les contraintes ne sont sans doute pas les mêmes en français et en anglais, car le paradigme des auxiliaires modaux n'est de fait pas équivalent. Néanmoins, dans les deux langues, il y a une corrélation entre les propriétés du discours en cours (son genre, sa fonction) et les propriétés distributionnelles d'une prédication d'existence qui met en scène une source énonciative qui n'est pas assimilable à un point de vue individuel. On peut ainsi poser que l'inversion à sujet nominal dans les deux langues encode une forme d'évidentialité qui neutralise la prise en charge du dire par le sujeténonciateur. 


\section{Bibliographie}

ATKInSON, J.-C., 1973, The Two Forms of Subject Inversion, The Hague, Paris, Mouton.

BAILARD, J., 1981, A Functional Approach to Subject Inversion, Studies in Language, 5, p. 1-29.

BIRNER, B. J., 1992, The Discourse Function of Inversion in English, Doctoral dissertation, Evanston, I11, Northwestern University.

BIRNER, B. J., 1994, «Information Status and Word Order: An Analysis of English Inversion », Language, 70, 2, p. 233-259

BOLINGER, D., 1977, Meaning and Form, London, Longman.

BORILlO, A., 2000, «Le complément locatif et le genre descriptif » in Coene, M., de Mulder, W., Dendale, P. \& D’Hulst,Y. (éds), Hommages à Liliane Tasmowski, Padoue, Unipress, p. 85-95.

BRESNAN, J., 1994, «Locative inversion and the architecture of the universal grammar » Language, 70(1), p. 72-131.

CHEN, R., 2003, English Inversion: A Ground-before-Figure Construction, New York, Mouton de Gruyter.

CoOpmans, P., 1989, «Where stylistic and syntactic processes meet: locative inversion in English », Language, 65, p. 728-751.

COPY C., GOURNAY, L., 2009, « Locative Inversion in Discourse: a Strategy of Non commitment » DISCOURS, 5 | 2009, [En ligne], mis en ligne le 04 novembre 2009, URL: http:// discours.revues.org/index 7355.html.

CORNISH, F., 2001, «L'Inversion locative en français, italien et anglais : propriétés syntaxiques, sémantiques et discursives », Les Cabiers de Grammaire, 26, p. 101-123.

Cornish, F., 2005, « A Cross-linguistic Study of so-called 'Locative Inversion': Evidence for the Functional Discourse Grammar model » in C. de Groot et K. Hengeveld (eds), Morphosyntactic Expression in Functional Grammar, Berlin/New York, Mouton de Gruyter, p. 163-202.

Cornish, F., 2008, «L'absence de prédication, le topique et le focus: le cas des phrases 'thétiques' », Faits de langues, 31-32, p. 121-131.

Culioli, A., 1990, Pour une linguistique de l'énonciation, t.1, Gap, Ophrys.

Culioli, A., 1999a, Pour une linguistique de l'énonciation, t.2, Gap, Ophrys.

Culioli, A., 1999b, Pour une linguistique de l'énonciation, t.3, Gap, Ophrys.

Dorgeloh, H., 1997, "Inversion in Modern English, Form and Function », SiDag, 6, Amsterdam, John Benjamins.

DorgeloH, H., 2006, « Inversion in descriptive narrative discourse: a text-typological account following functional principles », Cabiers de recherche, tome 9, p. 101-114.

Drubig, H., 1988, « On the Discourse Function of English Inversion » in J. Klegraf, D. Mehls (eds), Essays on the English Language and Applied Linguistics on the occasion of Gerhard Nickle's 60th birthday, Heidelberg, Groos. 
DufAYE, L., 2009, Théorie des opérations énonciatives et modélisation, Paris, Ophrys, Collection HDL.

DUfAYE, L., 2009, «CAN see / CAN hear. Quelques remarques sur la modalisation des verbes de perception » in Perception et structures linguistiques : buit études sur l'anglais, PUR.

FRANCKEL, J.-J., 2002, «Introduction à Le lexique, entre identité et variation », Langue Française, 133, p. 3-15.

FuCHS, C., 2006, «Locatif spatial initial et position du sujet nominal : pour une approche topologique de la construction de l'énoncé », Linguisticae Investigationes, 29 -1, p. 61-74.

Gournay, L., 2005, "Quand l'agencement est la trace d'une prédication 'objectivée' », Analele Universitatii din Craiova, Série Langues et littératures romanes, numéro spécial, IX, p. 65-74.

GOURNAY, L., 2006a, «Qu'est-ce qui distingue l'inversion absolue de l'inversion locative en français ? », Linguisticae Investigationes, 29 -1, p. 91-102.

GOURnAY, L., 2006b, Approche énonciative des catégories de marqueurs, Habilitation à Diriger des Recherches, Université Paris 7- Denis Diderot.

Gournay, L., 2007, « Verbal Constraints in Locative Inversions », Cabier de recherche, p. 57-68.

GOURNAY, L., 2009, «L'inversion locative : un cas particulier de prédication », Faits de langue, 30 \& 31, p. 263-276.

KorZEN, H., 1988, «Le classement des formes de l'inversion du sujet - dix-sept ans après » in M. Herslund, H. Korzen, G. Merad et J. Pedersen (eds), Traditions et tendances nowvelles des études romanes au Danemark. Articles publiés à l'occasion du 60e anniversaire d'Ebbe SpangHanssen, Etudes Romanes de l'Université de Copenhague, 31.

KorZEN, H., 1996, «L'unité prédicative et la place du sujet dans les constructions inversées », Langue Française, 111, p. 59-82.

KorZen, H., 2006, «L'anatomie de la construction bipartite. Petit essai sur l’inversion absolue et son contexte » in Nølke, H., Baron I., Korzen H., Korzen I. \& Müller H., Grammatica. Festschrift in honour of Michael Herslund, Bern, Peter Lang, p. 249-61.

Lahousse, K., 2003, The distribution of postverbal nominal subjects in French. Syntactic, semantic and pragmatic analyses, Thèse de Doctorat, Université Paris 10-Nanterre.

LAHOUSSE, K., 2011, Quand passent les cigognes, Presses universitaires de Vincennes.

LE BIDOIS, R., 1950, L'Inversion du sujet dans la prose contemporaine, Paris, Ed. d'Artrey.

LEVIN, B. \& RAPPAPORT HOVAV M., 1996, «Unaccusativity: at the syntax-lexical semantics interface », Linguistic Inquiry monograph, 26, The MIT Press.

Maingueneau, D., 2004, « Hyperénonciateur et 'particitation' », Langages, 156, p. 111-126.

MARANDIN, J.-M., 1999, «Unaccusative inversion in French » in Romance Languages and Linguistic Theory, p. 195-222.

MARANDIN, J.-M., 2003, «Inversion du sujet et structure de l'information dans les langues romanes » in Godard D. (ed.), Problèmes de la phrase simple, Langues Romanes, p. 345-392.

NAKAJIMA, H., 2001, «Verbs in Locative Constructions and the Generative Lexicon », The Linguistic Review, 17, p. 43-68.

QUIRK, R.. et al., 1972, A Comprehensive Grammar of the English Language, London, Longman. 
TASMOWSKI, L. \& WILLEMS, D., 1987, «Les phrases à première position actancielle vide : 'Par la porte ouverte (il) entrait une odeur de nuit et de fleurs ", Travaux de linguistique, 14-15, p. $177-192$.

WILLEMS, D., 1989, «Généricité, spécificité et constructions verbales : les structures à sujet inversé », Equivalence, 17-18, p. 175-181.

\section{Références des œuvres du corpus}

DAENINCKX, D., 1984, Meurtres pour mémoire, Gallimard.

DAHL, R., 2001, Tales of the Unexpected, Penguin, Recueil de nouvelles tirées de Kiss Kiss, 1960, et Someone like you, 1954.

D'Epernoux, F., 2001, Deux jours à tuer, Editions Anne Carrière.

Donaldson, J. \& SCHEFfler, A., 1999, The Gruffalo, Macmillan’s Childrens' Books.

EveTte, J.-B., 1997, Jordan Fantosme, NRF, Gallimard.

Hillerman, T., 1980, People of Darkness, Warner Books.

Hornby, N., 2001, How to be Good, Penguin, Viking.

JAMES, P.D., 1997, A Certain Justice, Ballantine Books.

JONQUeT, T., 2002, Ad vitam aeternam, Roman noir, Seuil.

LESSING, D., 1958, A Ripple from the Storm, Flamingo, 1993.

Malraux, A., 1946, La condition humaine, Gallimard, Folio, 1990.

Mirande, J., 1995, Contes et légendes du Moyen-Age, Nathan.

Morgan, R., 2000, Let There Be Lite, Bantam Books.

Mosley, W., 1991, A Red Death, Pan Books, 1993.

PAGAN, H., 1997, Dernière station avant l'autoroute, Rivages/Thriller.

PARETSKy, S., 1984, Deadlock, Penguin Books.

QuefFELEC, Y., 1985, Les Noces barbares, Folio, Gallimard

SNICKET, L., 2000, A Series of Unfortunate Events, The Wide Window, Egmont, New York.

VARGAS, F., 2002, Pars vite et reviens tard, Fleuve noir. 
